\title{
Reduced chatter sliding mode control for hydraulic manipulators based on continuous-time state dependent parameter models
}

\author{
H. Nemati, M. Bandala, O. L. R. Albrecht and C. J. Taylor \\ Engineering Department, Lancaster University, UK. Email: c.taylor@lancaster.ac.uk
}

\begin{abstract}
Sliding Mode Control (SMC) systems are developed for a hydraulic manipulator. The control model is obtained via state-dependent parameter (SDP) system identification. In contrast to previous research using discrete-time SDP models, in which the model coefficients are functions of the sampling interval, the present work develops a new continuous-time approach. It is well known that for conventional SMC there is a trade-off between chattering and robust performance. Hence, a recently developed approach to address this problem is investigated, in which the controller is designed via a fractional exponent of the sliding surface. The approach is developed for both conventional and Nonsingular Terminal SMC (NTSMC). The new continuous version of the NTSMC algorithm successfully reduces chattering and provides the best overall performance of various SMC designs. However, for the preliminary experiments reported in this article, a PID lead-lag controller yields the lowest absolute errors, albeit at the cost of a higher control effort. Hence, given that dead-zone and other uncertainties provide the main motivation for use of SMC in this application, further research into the robustness of the new algorithm is required.

Index Terms-sliding mode control; state-dependent parameter model; continuous-time; hydraulic manipulator
\end{abstract}

\section{INTRODUCTION}

This article concerns state dependent parameter (SDP) modelling and sliding mode control (SMC) design for hydraulic manipulators. The research is motivated by the authors' work on assisted tele-operation for the decommissioning and characterisation of nuclear environments, for which improved control systems are sometimes required [1]-[5]. Hence, the experimental results are based on a recently reconfigured, hydraulically-actuated, dual-manipulator device in the laboratory. In contrast to the earlier articles, the present work investigates a continuous-time SDP model and utilises this for nonsingular terminal SMC of joint angle.

Wang et al. [6] and Wind et al. [7] have proposed new methods to estimate joint angle, velocity and acceleration of hydraulic manipulators, without considering nonlinearities such as actuator dead-zones. In fact, the two major challenges in high performance positioning and tracking stabilisation of robot manipulators are the friction between moving parts and the dead-zone of the actuators. Friction may lead to stick slip and steady-state error while dead-zones may severely

This work is supported by the UK Engineering and Physical Sciences Research Council (EPSRC) grant EP/R02572X/1. limit system performance [8]. This is more challenging for hydraulic driving systems, in which the dead-zone phenomena are time-varying and involve unknown characteristics. In this context, Huang et al. [9] employ an incremental nonlinear dynamic inversion (INDI) strategy to enhance the robustness of conventional NDI approaches [10]. However, the dead-zone problem was not addressed in that study and the stability of INDI in the presence of external disturbances remains an open problem. More generally, INDI can suffer from measurement and actuator delays. Xu and Yoon [11] describe use of a PID controller to stabilise hydraulic manipulators via vision-based estimation of the pose. However, the main disadvantage of the image-based approach is the high sensitivity to action change, and view and size variability.

Sliding mode control (SMC) has long been recognised as a robust nonlinear control methodology because of its strong stability, and low sensitivity to model parameter variations and external disturbances. SMC is designed such that the system trajectory is attracted by a predetermined manifold $(\sigma)$ in the phase plane. Once it reaches $\sigma$, the trajectory subsequently slides along it. One limitation of conventional SMC is that tracking errors do not converge to zero in a finite time. To enhance the convergence performance, terminal sliding mode control (TSMC) has been introduced: see for example references [12] and [13]. However, TSMC can suffer from singularity problems due to the negative fractional exponent in the terminal sliders. Nonsingular terminal sliding mode control (NTSMC) addresses this problem [14]-[18].

However, despite the popularity of conventional SMC, the chattering problem remains a major drawback, discouraging its direct use. One solution is to use a continuous approximation of the switching element (known as the boundary layer) instead of the signum function. Rubio [19], for example, applies conventional SMC to a robot manipulator with deadzone nonlinearities, based on this boundary layer method. Cheong et al. [20] introduce an adaptive fuzzy sliding mode observer-based position controller to compensate friction and the dead-zone of a robot manipulator, again using the boundary layer method. However, use of the boundary layer method has a critical problem: the system performance highly depends upon the boundary layer thickness.

To address these issues, the present research employs 
a chatter-free approach developed independently by [21] and [22], in which the controller is designed through the use of a fractional exponent of the sliding surface. In the present article, this approach is adapted for both conventional SMC and, to ensure finite time stability, a novel continuous and smooth NTSMC algorithm. Note that while Du et al. [22] consider 'discrete-time' chatter-free SMC, the present authors use a 'continuous-time' version of a similar concept. Furthermore, Du et al. [22] do not consider NTSMC, hence one contribution of the present article is to illustrate the flexibility of the continuous method, which can in principle be applied to any SMC technique. Several SMC design options, together with an industrial PID controller, are experimentally compared.

A second contribution concerns the SDP modelling. The parameters of SDP models are functionally dependent on measured variables, such as joint angles and velocities in the case of manipulators, normally defined in discrete-time terms [23]-[25]. By contrast, the results below utilise a continuous-time SDP model that is not dependent on the sampling interval and which takes a suitable form for SMC. To the authors' knowledge, this represents the first time that SDP models have been used to design SMC systems. Finally, the manipulators used for the experimental results have recently been reconfigured (in comparison to the earlier cited work at Lancaster University), both in terms of the hardware framework and the data logging and control software, hence new model coefficients are required.

Thus, the novel research contributions (and organisation) of the article concern the reconfigured hardware (section II); the development of a continuous-time SDP modelling approach for hydraulic manipulators (section III); the design of continuous chatter-free SMC algorithms based on this model (section IV); and, finally, a comparative experimental investigation into the various new and conventional control schemes (section $\mathrm{V}$ ). The conclusions are presented in section VI.

\section{LABORATORY SYSTEM}

The laboratory demonstrator used in this article consists of two HYDROLEK HLK-7W manipulators, each a 6-degreesof-freedom articulated arm, with a seventh actuator for the gripper, as illustrated by Fig. 1. Whilst the original setup is described by e.g. reference [1], a ball valve, pressure gauge and new pressure pump were added in 2019. Fig. 2 shows the location of these new elements. The hydraulic system was upgraded with a Bosch Rexroth Pressure \& Tank Circuit Hydraulic Power Unit (MKPTO415V16V15). This provides $5.5 \mathrm{~L} / \mathrm{min}$ at 220 bar and has a 15 litre oil tank.

The manipulators are now controlled via a NI Compact DAQ 9132 system. The cDAQ 9132 is a $1.33 \mathrm{GHz}$ Dual-core atom computer with 4 slots for I/O modules. The system runs both Windows 7 Embedded Edition and Labview 2018 for programming and interfacing. The cDAQ 9132 utilises three I/O modules: one NI 9205 i.e. a 32-channel analogue-to-digital (ADC) converter and two NI 9264 i.e. 16-channel digital-toanalogue converters (DAC). The two NI 9264 modules are used to actuate the P02AD1 valves in the two manipulators.
Finally, the position angle sensors are standard rotary linear potentiometers.

A dedicated box is installed next to the robot in order to hold the controller and associated equipment. A monitor, mouse and keyboard are externally connected such that an operator can control or program the robot from outside the safety cell (to some degree, representing the situation on a nuclear site where the robot will be remote from the operator). Since the present article focuses on the low-level joint control problem, inverse kinematics and the human-machine interface are not described here: see e.g. [5] for a recent reference.

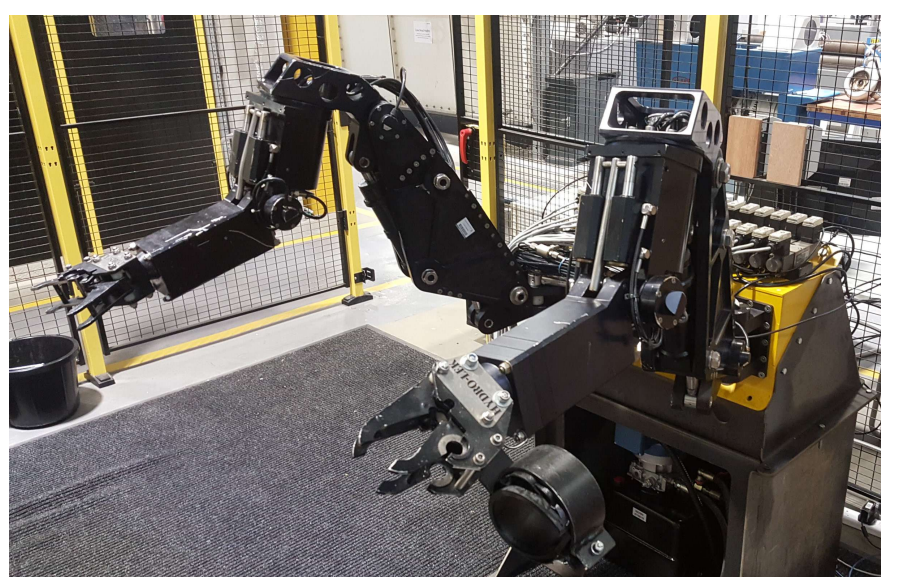

Fig. 1. HYDROLEK HLK-7W manipulators in the laboratory.

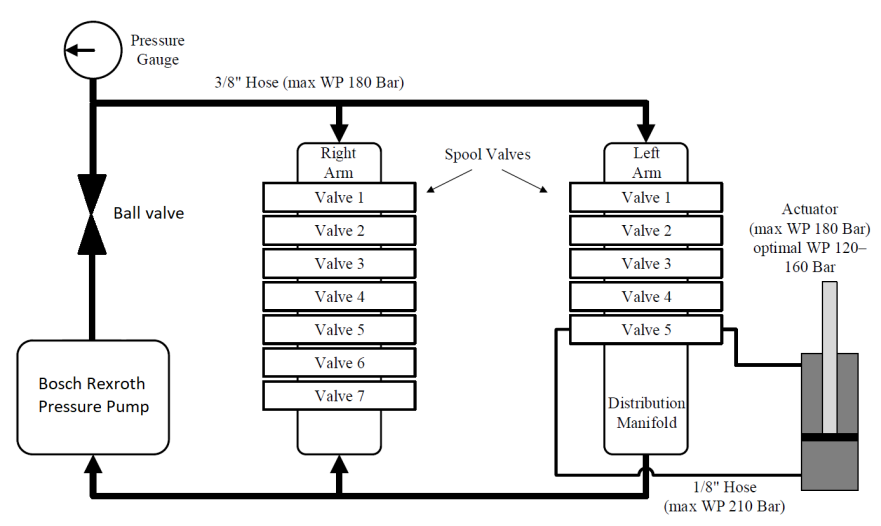

Fig. 2. Schematic diagram of the reconfigured hydraulic system.

\section{SYSTEM IDENTIFICATION}

The deterministic discrete-time SDP model is often expressed as $y_{k}=\mathbf{w}_{k}^{\mathrm{T}} \mathbf{p}_{k}$, where the SDP parameter vector is defined,

$$
\mathbf{p}_{k}=\left[\begin{array}{llllll}
a_{1}\left\{\chi_{k}\right\} & \cdots & a_{n}\left\{\chi_{k}\right\} & b_{1}\left\{\chi_{k}\right\} & \cdots & b_{m}\left\{\chi_{k}\right\}
\end{array}\right]^{\mathrm{T}}
$$

in which $a_{i}\left\{\chi_{k}\right\}$ and $b_{j}\left\{\chi_{k}\right\}$ are $n$ and $m$ state dependent parameters i.e. functions of a non-minimal state vector $\chi_{k}$,

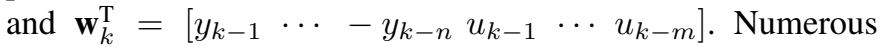
publications discuss how to estimate this model from data, for example using an iterative backfitting algorithm implemented 
in stochastic state space form [26], [27]. The present authors use the CAPTAIN toolbox for MATLAB [28].

For brevity, details of these methods are omitted from the present article, which instead focuses on SMC design. However, a brief overview follows, in particular to emphasise the continuous-time formulation introduced here and the way it is adapted for the specific case of hydraulically-actuated manipulators. In this case, $u_{k}$ and $y_{k}$ are replaced by $u(t)$ and $\theta(t)$ respectively, representing the control input and joint angle, and noting that $u(t)$ is a scaled signal in the range \pm 10 i.e. the applied voltage for the two directions of movement. The SDP hydraulic manipulator model is subsequently identified in three stages as follows:

Step 1. Open-loop step experiments, such as those shown in Fig. 3, suggest that a first order linear differential equation,

$$
\dot{\theta}(t)=-a_{1} \theta(t)+b_{1} u(t-\tau)
$$

provides an approximate representation of individual joints, with $a_{1}, b_{1}$ and the time-delay $\tau$ estimated using the SRIVC algorithm in the CAPTAIN toolbox. Illustrative model responses and experimental data are shown in Fig. 3.

Step 2. However, the model (1) is only valid for straightforward step experiments, and even in this case the values of $\left\{a_{1}, b_{1}\right\}$ are not repeatable for experiments with different input magnitudes. In fact, further analysis of experimental data using SDP methods, suggests that $a_{1} \approx 0$ is time invariant, whilst the single state dependent parameter is a function of the lagged input signal i.e. $b_{1}\{\chi(t)\}=b_{1}\{u(t-\tau)\}$. The mechanistic interpretation of this model is straightforward: $b_{1}\{u(t)\} \times u(t)$ directly represents the angular velocity. Hence, defining $q\{u(t)\}=b_{1}\{u(t)\} \times u(t)$, the model is,

$$
\dot{\theta}(t)=q\{u(t-\tau)\}
$$

where $q\{u(t-\tau)\}$ represents a static nonlinear function of the input. To illustrate using the right hand side 'slew' joint for horizontal movement of the manipulator, denoted J1, with $\Delta t=0.01 \mathrm{~s}$ and $\tau=0.22 \mathrm{~s}$, SRIVC estimates $b_{\tau}$ are determined from Fig. 3 and plotted as the circles in Fig. 4, which highlights the dead-zone and the velocity saturation.

Step 3. Finally, $q\{u(t-\tau)\}$ is parameterized and estimated directly from experimental data (including from experiments that excite the system at a wider range of frequencies and magnitudes; for brevity, not plotted). For Joint 1, this yields,

$q\{u(t)\}= \begin{cases}\alpha_{1}-\alpha_{1} e^{-\alpha_{2}\left(\alpha_{3}-u(t)\right)} & \text { for } u(t)<\alpha_{3} \\ 0 & \text { for } \alpha_{3} \leq u(t) \leq \alpha_{6} \\ \alpha_{4}-\alpha_{4} e^{-\alpha_{5}\left(u(t)-\alpha_{6}\right)} & \text { for } u(t)>\alpha_{6}\end{cases}$

where $\hat{\alpha}_{i}=[-25.19,16.02,-1.34,29.89,8.55,1.28]$. To obtain these estimates, experimental data are compared with the model response and the mean sum of the least squares output errors are used as the objective function for numerical optimisation in MATLAB. It is clear that $\left(\alpha_{2}, \alpha_{5}\right)$ are curve coefficients; $\alpha_{1}=-25.19^{\circ} \mathrm{s}^{-1}$ and $\alpha_{4}=29.89^{\circ} \mathrm{s}^{-1}$ provide minimum and maximum angular velocity saturation limits; and
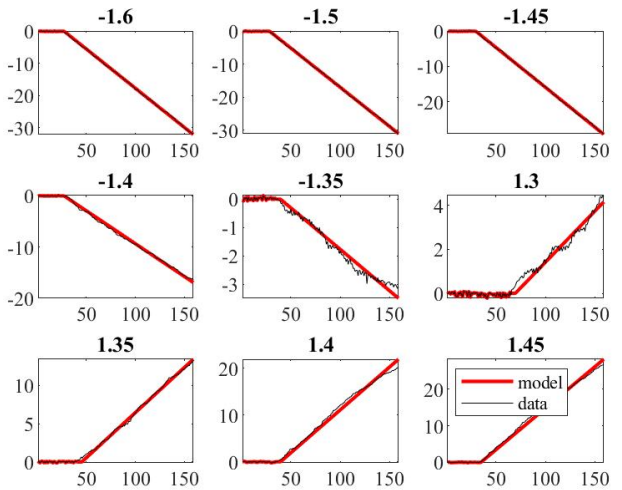

Fig. 3. Open-loop experiments for Joint 1 using step inputs for a range of magnitudes $(-1.6,-1.5$ etc.) and response of the linear model (1).

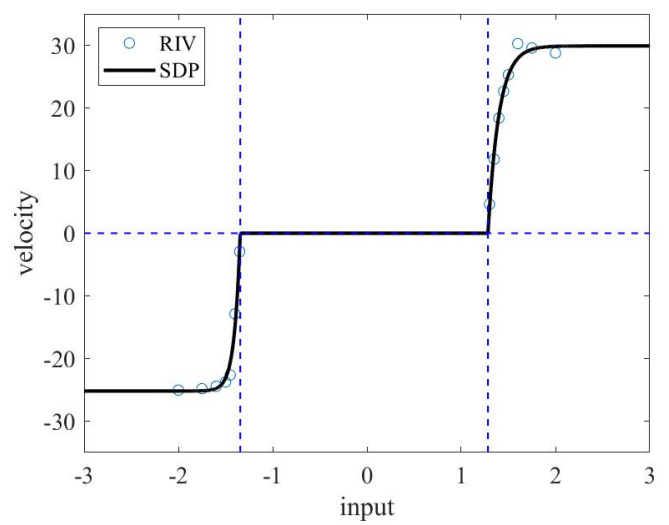

Fig. 4. Angular velocity plotted against input magnitude (i.e. steady state voltage), showing SRIVC estimates $b_{1} \times u(t)$ for individual experiments (circles) and optimised $q\{u(t)\}$. The dead-zone is highlighted (dashed).

$\alpha_{3}=-1.34 \mathrm{~V}$ to $\alpha_{6}=1.28 \mathrm{~V}$ is the dead-zone. These objective estimates compare closely with equivalent values obtained from extensive prior ad hoc experimental work. Finally, the nonlinear model has constraints $\theta_{\min }<\theta(t)<\theta_{\max }$, where $\theta_{\min }=-27^{\circ}$ and $\theta_{\max }=40^{\circ}$ are the hardware limits for $\mathrm{J} 1$.

Similar SDP model forms are successfully identified for each joint of both manipulators, although the preliminary results for SMC considered below focus on control of $\mathrm{J} 1$ in isolation. In this regard, it should be pointed out that although Eq. (2) represents the hydraulic manipulator model identified from data, for SMC design the above coefficients are sometimes empirically adjusted to obtain satisfactory closedloop responses (see below). Furthermore, for the initial results below, SMC design assumes $\dot{\theta}(t)=q\{u(t)\}$ i.e. $\tau=0$.

\section{Control Design}

The various SMC approaches are developed. 


\section{A. Conventional Sliding Mode Control}

A conventional integral sliding surface can be expressed in scalar form as follows,

$$
\sigma_{\text {conv }}=\tilde{x}+\lambda \int \tilde{x} d t
$$

where $\tilde{x}=x-x_{d}$ is the error term, i.e. the difference between the system output and the desired trajectory, and $\lambda$ is a positive constant. To guarantee the stability of the sliding surface, consider the following positive definite function of $\sigma_{\text {conv }}$,

$$
V=\frac{1}{2} \sigma_{c o n v}^{2}
$$

According to the second method of Lyapunov, the timederivative of Eq. (4) must satisfy $\dot{V}<0$, i.e.,

$$
\dot{V}=\sigma_{\text {conv }}(\dot{\tilde{x}}+\lambda \tilde{x})
$$

Therefore,

$$
\sigma_{\text {conv }}(\dot{\tilde{x}}+\lambda \tilde{x})=-\eta\left|\sigma_{\text {conv }}\right|
$$

and,

$$
\dot{\tilde{x}}+\lambda \tilde{x}=-\eta \operatorname{sign}\left(\sigma_{\text {conv }}\right)
$$

In Eq. (7), $\eta$ is a positive constant and $\operatorname{sign}\left(\sigma_{\text {conv }}\right)$ is a discontinuous signum function defined as,

$$
\operatorname{sign}\left(\sigma_{\text {conv }}\right)= \begin{cases}-1 & \sigma_{\text {conv }}<0 \\ 0 & \sigma_{\text {conv }}=0 \\ +1 & \sigma_{\text {conv }}>0\end{cases}
$$

Substituting Eq. (2) into Eq. (7) yields,

$$
u_{\text {conv }}= \begin{cases}u_{1, \text { conv }} & v<\alpha_{3} \\ 0 & \alpha_{3} \leq v \leq \alpha_{6} \\ u_{2, \text { conv } v} & v>\alpha_{6}\end{cases}
$$

where,

$$
\begin{aligned}
u_{1, \text { conv }}= & \alpha_{3}+ \\
& \frac{\ln \left[\frac{1}{\alpha_{1}}\left(\alpha_{1}-\dot{\theta}_{d}+\lambda\left(\theta-\theta_{d}\right)\right)+\eta \operatorname{sign}\left(\sigma_{\text {conv }}\right)\right]}{\alpha_{2}} \\
u_{2, \text { conv }}= & \alpha_{6}- \\
& \frac{\ln \left[\frac{1}{\alpha_{4}}\left(\alpha_{4}-\dot{\theta}_{d}+\lambda\left(\theta-\theta_{d}\right)\right)+\eta \operatorname{sign}\left(\sigma_{\text {conv }}\right)\right]}{\alpha_{5}}
\end{aligned}
$$

Finally, to minimise the chattering phenomenon that often arises in conventional SMC, the Boundary Layer (BL) approach is employed, in which $\operatorname{sign}\left(\sigma_{c o n v}\right)$ is replaced by $\tanh \left(\sigma_{c o n v}\right)$ in Eqs. (10) and (11). For the results discussed in section $\mathrm{V}$, this is referred to as conventional SMC-BL design.

\section{B. Nonsingular Terminal Sliding Mode Control}

Use of a nonlinear sliding surface can improve the performance of conventional SMC by assuring finite time convergence. Without loss of generality, the nonsingular terminal sliding surface is represented as,

$$
\sigma_{N T S M C}=\int \tilde{x} d t+\frac{1}{\lambda} \tilde{x}^{\frac{q}{p}}
$$

where $p$ and $q$ are positive odd integers $(p<q<2 p)$. In a similar manner to Eq. (4) and Eq. (9), using the second method of Lyapunov, if the nonsingular terminal sliding surface is Eq. (12), then the NTSM controller is derived as follows,

$$
u_{N T S M C}= \begin{cases}u_{1, N T S M C} & v<\alpha_{3} \\ 0 & \alpha_{3} \leq v \leq \alpha_{6} \\ u_{2, N T S M C} & v>\alpha_{6}\end{cases}
$$

where,

$$
\begin{aligned}
& u_{1, N T S M C}=\alpha_{3}+\frac{\ln \left[g_{1}\right]}{\alpha_{2}} \\
& u_{2, N T S M C}=\alpha_{6}-\frac{\ln \left[g_{2}\right]}{\alpha_{5}}
\end{aligned}
$$

in which,

$g_{1}=\frac{1}{\alpha_{1}}\left(\alpha_{1}-\dot{\theta}_{d}+\frac{p \lambda}{q}\left(\left(\theta-\theta_{d}\right)^{2-\frac{q}{p}}+\eta \operatorname{sign}\left(\sigma_{N T S M C}\right)\right)\right)$ $g_{2}=\frac{1}{\alpha_{4}}\left(\alpha_{4}-\dot{\theta}_{d}+\frac{p \lambda}{q}\left(\left(\theta-\theta_{d}\right)^{2-\frac{q}{p}}+\eta \operatorname{sign}\left(\sigma_{N T S M C}\right)\right)\right)$

Again, to minimise the chattering phenomenon, $\operatorname{sign}\left(\sigma_{N T S M C}\right)$ is replaced by $\tanh \left(\sigma_{N T S M C}\right)$ in Eqs. (14) and (15). This is referred to as NTSMC-BL design.

\section{Continuous Sliding Mode Control}

Discontinuous control laws are generally incompatible with many systems that require continuous controllers. As noted in section I, early SMC theory used continuous controllers with a continuous function (e.g. hyperbolic or saturation) in the vicinity of the sliding surface. However, zero tracking error is not ensured within a finite time. Furthermore, usually there is a direct trade-off between chattering and robust performance i.e. chattering reduction may lead to reduced robustness. In the present subsection, a continuous and smooth controller approach is utilised to address this problem i.e. a fractional exponent of the sliding surface is used to minimise chattering whilst retaining robust performance. The approach is applied to both conventional SMC and NTSMC to ensure finite time stability and singularity avoidance. In the latter case, Eqs. (14) and (15) become,

$$
\begin{aligned}
& u_{1, C N T S M C}=\alpha_{3}+\frac{\ln \left[g_{3}\right]}{\alpha_{2}} \\
& u_{2, C N T S M C}=\alpha_{6}-\frac{\ln \left[g_{4}\right]}{\alpha_{5}}
\end{aligned}
$$

in which,

$$
g_{3}=\frac{1}{\alpha_{1}}\left(\alpha_{1}-\dot{\theta}_{d}+\frac{p \lambda}{q}\left(\left(\theta-\theta_{d}\right)^{2-\frac{q}{p}}+\eta \sigma_{C N T S M C}{ }^{\frac{m}{n}}\right)\right)
$$


$g_{4}=\frac{1}{\alpha_{4}}\left(\alpha_{4}-\dot{\theta}_{d}+\frac{p \lambda}{q}\left(\left(\theta-\theta_{d}\right)^{2-\frac{q}{p}}+\eta \sigma_{C N T S M C} C^{\frac{m}{n}}\right)\right)$

where $m$ and $n$ are positive odd integers $(0<m<n)$. It should be emphasised that this approach can be applied to any SMC strategy not only NTSMC design.

\section{EXPERIMENTAL RESULTS}

The SMC approaches are compared with a PID controller with lead compensation, optimised using standard NI Labview tools. The five different controllers, including PID, conventional SMC-BL, NTSMC-BL, continuous SMC and continuous NTSMC are experimentally applied to J1 of the hydraulic manipulator. The initial condition $\theta(0)=20^{\circ}$, while the control gains are selected by trial and error experimentation: $\left[\begin{array}{ll}K & T_{\text {lead }}\end{array}\right]=\left[\begin{array}{ll}1 & 10^{-3}\end{array}\right]$ and $\left[\begin{array}{ll}\lambda & \eta\end{array}\right]=$ $10^{-3}\left[\begin{array}{ll}5 & 1\end{array}\right]$. The fractional exponents in NTSMC-BL and continuous NTSMC are $q=n=5$ and $p=m=3$.

Figs. 5 and 6 show the joint angle time history for the various control schemes, whilst Table I summarises the performance in terms of an Integral of Absolute Error (IAE) criterion i.e. for deviation of the angle from the set point. Table I shows that the IAE value of conventional SMC-BL is much greater than that of the other controllers, because the output is oscillating around the desired trajectory (Fig. 5). The new continuous-time NTSMC approach has the smoothest response and lowest IAE of the various SMC approaches, demonstrating the potential advantage of this concept. However, it is also clear that, for this limited experiment, NTSMC offers no benefit over a conventional PID lead-lag design. Indeed, the IOE of the PID controller is lower than continuous-time NTSMC, albeit at the expense of a higher control effort.

In the latter regard, the time history of the control input is illustrated in Figs. 7 and 8. These results show that the control response has high frequency oscillations over time for both PID and conventional SMC-BL controllers, whilst Fig. 8 demonstrates that the terminal slider and both continuous approaches successfully reduce control effort. Finally, Table I also compares the control efforts based on the Root-MeanSquare (RMS) of the executed voltages for the aforementioned controllers, showing that the consumed energy for PID is higher than for the continuous NTSMC scheme.

\section{CONCLUSIONS}

Five schemes have been compared for control of a hydraulic manipulator in the laboratory, including PID, conventional SMC, Nonsingular Terminal SMC and continuous-time versions of both SMC and NTSMC. Experimental results show

TABLE I

CONTROL PERFormance

\begin{tabular}{|c|c|c|}
\hline Controllers & IAE (deg.s) & RMS (V) \\
\hline PID (Lead) & 20.9916 & 1.3161 \\
\hline SMC-BL & 46.7869 & 2.2990 \\
\hline NTSMC-BL & 25.0219 & 0.7929 \\
\hline Continuous SMC & 34.8450 & 0.8397 \\
\hline Continuous NTSMC & 23.8945 & 0.9518 \\
\hline
\end{tabular}

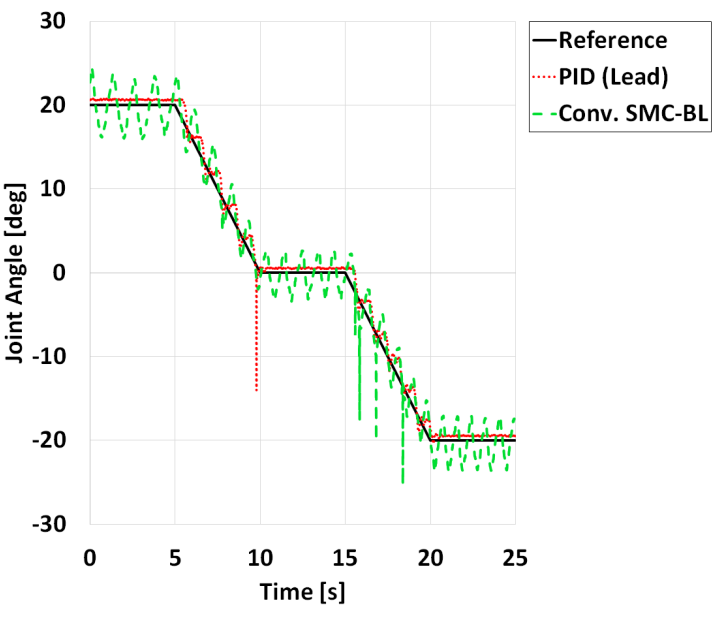

Fig. 5. Joint angle time history for PID and conventional SMC-BL.

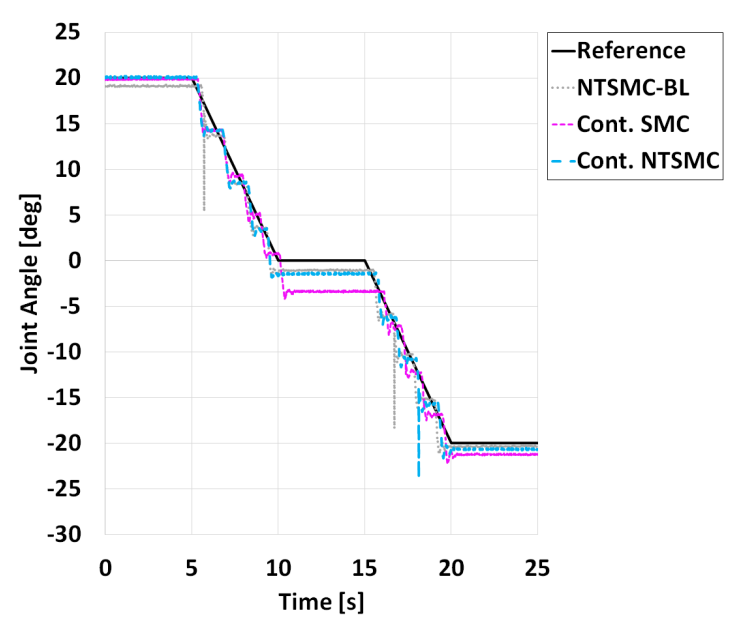

Fig. 6. Joint angle for NTSMC-BL and continuous SMC/NTSMC.

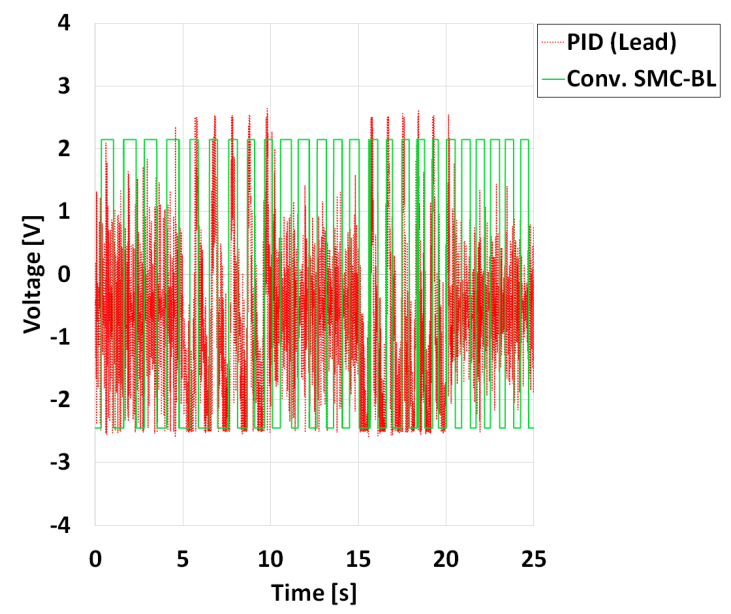

Fig. 7. Control input time history for PID and conventional SMC-BL. 


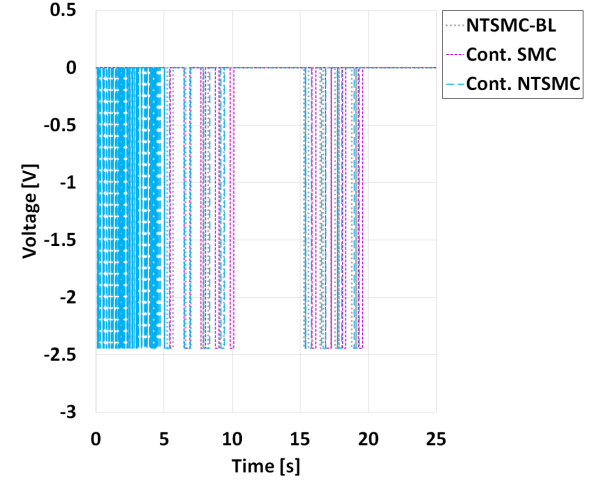

Fig. 8. Control input for NTSMC-BL and continuous SMC/NTSMC.

that the novel continuous NTSMC approach successfully enhances the system performance whilst reducing chattering, in comparison to the other SMC approaches. However, it is true to say that the preliminary experimental results reported in this article are disappointing in comparison to the benchmark PID lead-lag controller. The authors are presently working on the various issues raised and these results will be reported in future publications. Hence, ongoing research includes:

- Given that dead-zone and other uncertainties provide the main motivation for use of SMC in this application, a wider range of experimental scenarios and further research into the robustness of the new algorithm is required, to more fully evaluate its potential for hydraulic manipulator control e.g. external disturbances.

- These experiments utilise all seven DoF for tasks in connection to, for example, pipe cutting [5] and radiological scanning [29], for nuclear decommissioning.

- Further investigations into the way the model is constructed, including comparisons between the SDP system identification approach and conventional mechanistic models for the robot are required. Similar applies to methods for implementing the continuous-time designs in software, given practical processing and other constraints.

\section{REFERENCES}

[1] A. Montazeri, C. West, S. Monk, and C. J. Taylor, "Dynamic modeling and parameter estimation of a hydraulic robot manipulator using a multiobjective genetic algorithm," International Journal of Control, vol. 90, no. 4 , pp. 661-683, 2017.

[2] T. Burrell, C. West, S. Monk, A. Montazeri, and C. J. Taylor, "Towards a cooperative robotic system for autonomous pipe cutting in nuclear decommissioning," in 12th UKACC International Conference on Control, Sheffield, UK, September 2018.

[3] C. West, S. Monk, A. Montazeri, and C. J. Taylor, "A vision-based positioning system with inverse dead-zone control for dual-hydraulic manipulators," in 12th UKACC International Conference on Control, Sheffield, UK, September 2018.

[4] I. Tsitsimpelis, C. J. Taylor, B. Lennox, and M. J. Joyce, "A review of ground-based robotic systems for the characterization of nuclear environments," Prog. Nuclear Energy, vol. 111, pp. 109-124, 2019.

[5] M. Bandala, C. West, S. Monk, A. Montazeri, and C. J. Taylor, "Visionbased assisted tele-operation of a dual-arm hydraulically actuated robot for pipe cutting and grasping in nuclear environments," Robotics, vol. 8, no. 6: 42, pp. 1-24, 2019.
[6] W. Wang, H. Chi, S. Zhao, and Z. Du, "A control method for hydraulic manipulators in automatic emulsion filling," Automation in Construction, vol. 91, pp. 92-99, 2018.

[7] H. Wind, A. Renner, S. Schaut, S. Albrecht, and O. Sawodny, "Comparison of joint angle, velocity and acceleration estimators for hydraulically actuated manipulators to a novel dynamical approach," Control Engineering Practice, vol. 91, pp. 104-118, 2019.

[8] G. Tao and T. V. Kokotovic, "Adaptive control of plants with unknown dead-zones," IEEE Trans. Aut. Control, vol. 39, no. 1, pp. 59-68, 1994.

[9] H. Deng, J. Luo, X. Duan, and G. Zhong, "Adaptive inverse control for gripper rotating system in heavy-duty manipulators with unknown deadzones," IEEE Trans. Ind. Elect., vol. 64, pp. 7952-7961, 2017.

[10] Y. Huang, D. M. Pool, O. Stroosma, and Q. Chu, "Long-stroke hydraulic robot motion control with incremental nonlinear dynamic inversion,' IEEE/ASME Trans. Mechatronics, vol. 24, no. 1, pp. 304-314, 2019.

[11] J. Xu and H.-S. Yoon, "Vision-based estimation of excavator manipulator pose for automated grading control," Automation in Construction, vol. 98, pp. 122-131, 2019.

[12] S. I. Han and J. Lee, "Finite time sliding surface constrained control for a robot manipulator with an unknown deadzone and disturbance," ISA Transactions, vol. 65, pp. 307-318, 2016.

[13] J. Kim, M. Jin, W. Choi, and J. Lee, "Discrete time delay control for hydraulic excavator motion control with terminal sliding mode control," Mechatronics, vol. 60, pp. 15-25, 2019.

[14] Y. Feng, X. Yu, and Z. Man, "Non-singular terminal SMC of rigid manipulators," Automatica, vol. 38, pp. 2159-2167, 2002.

[15] M. D. Tran and H. J. Kang, "Non-singular terminal sliding mode control of uncertain second-order nonlinear systems," Mathematical Problems in Engineering, 2015. ID: 181737.

[16] H. Wang, L. Shi, Z. Man, J. Zheng, S. Li, M. Yu, C. Jiang, H. Kong, and Z. Cao, "Continuous fast nonsingular terminal sliding mode control of automotive electronic throttle systems using finite-time exact observer," IEEE Trans. Industrial Electronics, vol. 65, pp. 7160-7172, 2016.

[17] X. Yu and M. Zhihong, "Fast terminal sliding-mode control design for nonlinear dynamical systems," IEEE Trans. Circuits and Systems I: Fundamental Theory and Applications, vol. 49, pp. 261-264, 2002.

[18] T. X. Dinh, T. D. Thien, T. H. V. Anh, and K. K. Ahn, "Disturbance observer based finite time trajectory tracking control for a 3 dof hydraulic manipulator including actuator dynamics," IEEE Access, vol. 6 , pp. 36798-36809, 2018

[19] J. d. J. Rubio, "Sliding mode control of robotic arms with deadzone," IET Control Theory and App., vol. 11, no. 8, pp. 1214-1221, 2017.

[20] J. Y. Cheong, S. I. Han, and J. M. Lee, "Adaptive fuzzy dynamic surface sliding mode position control for a robot manipulator with friction and deadzone," Mathematical Problems in Eng., vol. 2013, pp. 1-15, 2013.

[21] H. Nemati, M. Oishi, N. Kobayashi, R. Nakata, and S. Hokamoto, "New continuous sliding mode design for spacecraft attitude control," in 24th Workshop on JAXA Astrodynamics and Flight Mechanics, Sagamihara, Japan, July 2014.

[22] H. Du, X. Yu, M. Z. Q. Chen, and S. Li, "Chattering-free discrete-time sliding mode control," Automatica, vol. 68, pp. 87-91, 2016.

[23] C. J. Taylor, E. M. Shaban, M. A. Stables, and S. Ako, "Proportionalintegral-plus control applications of state-dependent parameter models,' IMECHE Proceedings Part I: Journal of Systems and Control Engineering, vol. 221, no. 17, pp. 1019-1032, 2007.

[24] C. J. Taylor and D. Robertson, "State-dependent control of a hydraulically-actuated nuclear decommissioning robot," Control Engineering Practice, vol. 21, no. 12, pp. 1716-1725, 2013.

[25] A. Janot, P. C. Young, and M. Gautier, "Identification and control of electro-mechanical systems using state-dependent parameter estimation," International Journal of Control, vol. 90, pp. 643-660, 2017.

[26] P. C. Young, Recursive Estimation and Time Series Analysis: An Introduction for the Student and Practitioner. Springer, 2011.

[27] C. J. Taylor, P. C. Young, and A. Chotai, True Digital Control: Statistical Modelling and Non-Minimal State Space Design. Wiley, 2013.

[28] C. J. Taylor, P. C. Young, W. Tych, and E. D. Wilson, "New developments in the CAPTAIN Toolbox for Matlab with case study examples," in 18th IFAC Symposium on System Identification (SYSID), Stockholm, Sweden, July 2018.

[29] S. Monk, M. Bandala, D. Cheneler, A. Montazeri, N. Dixon, C. West, and C. Taylor, "COTS vision system, radiation sensor and 6 DoF robot for use in identifying radiologically uncharacterised objects," in 4th Australian and New Zealand Control Conference (ANZCC), Gold Coast, Australia, November 2020. 